\title{
In situ removal and purification of biosurfactants by automated surface enrichment
}

\author{
Julia Glazyrina $\cdot$ Stefan Junne $\cdot$ Peter Thiesen • \\ Klaus Lunkenheimer • Peter Goetz
}

Received: 16 June 2008 /Revised: 10 July 2008 / Accepted: 11 July 2008 /Published online: 9 August 2008

(C) The Author(s) 2008. This article is published with open access at Springerlink.com

\begin{abstract}
A new method is described to remove and separate biosurfactants from complex mixtures by compressing and harvesting the liquid surface layer. This method was applied to Bacillus subtilis cultures, in which the lipopeptide antibiotic fengycin as well as the polyketide antibiotic bacillaene were produced. The automated harvesting and collection in a custom-built glass body called 'flounder' was repeated several hundred times. The fengycin concentration in the fractions was found to be four times higher than in the culture centrifugate. Of the overall fengycin, $50 \%(w / w)$ were recovered after 300 cycles, $95 \%(w / w)$ after 800 harvesting cycles. A separation of fengycin from the less surface-active bacillaene could be achieved due to stronger surface activity of fengycin. The ratio of partition coefficients of fengycin and bacillaene was
\end{abstract}

Julia Glazyrina and Stefan Junne contributed equally to this work.

P. Goetz $(\bowtie)$

Institut für Bioverfahrenstechnik, Universität Stuttgart,

Allmandring 31,

70569 Stuttgart, Germany

e-mail: Peter.Goetz@ibvt.uni-stuttgart.de

J. Glazyrina $\cdot$ S. Junne

Institut für Biotechnologie, Fachgebiet Bioverfahrenstechnik,

Technische Universität Berlin,

Ackerstraße 71-76, ACK24,

13355 Berlin, Germany

P. Thiesen

Nanofilm Surface Analysis, Halcyonics GmbH,

Anna-Vandenhoeck-Ring 5,

37081 Goettingen, Germany

K. Lunkenheimer

Max-Planck-Institute of Colloids and Interfaces,

Am Mühlberg 1,

14424 Potsdam-Golm, Germany nine times higher compared to foam fractionation. A stepwise increase of the equilibrium surface tension in the centrifugate from 29 to $33 \mathrm{mN} / \mathrm{m}$ indicated a fractionated separation of different surface-active substances. The utilization of cell containing culture broth instead of centrifugate had only slight effects on separation efficiency. These results demonstrate the possibility to separate biosurfactants directly from cultivation without the use of extraction solvents or foam formation.

Keywords Biosurfactant - Separation · Bacillus subtilis . Antibiotic $\cdot$ Fengycin $\cdot$ Bacillaene

\section{Introduction}

Biosurfactants are amphiphilic surface-active substances of microbial origin. Main classes of biosurfactants are glycolipids, lipopeptides, phospholipids, and fatty acids (Banat et al. 2000). They are present on the cell surface or excreted extracellularly. Biosurfactants are of rising interest, since they are often less toxic than chemically synthesized surfactants (Makkar and Cameotra 2002). Biosurfactants are showing extraordinary strong surface activities. For example, surfactin, which is a lipopeptide synthesized by Bacillus subtilis, lowers the surface tension of water from 72 to $27 \mathrm{mN} / \mathrm{m}$ at a concentration of as low as $20 \mu \mathrm{M}$ (Peypoux et al. 1999).

One limitation in biosurfactant production is their inhibitory effect on their own synthesis. It could be shown that two-phase partitioning systems with in situ extraction of the surfactant-enriched phase can accelerate biosurfactant production (Drouin and Cooper 1992; Kuboi et al. 1994). Other recovery methods that can be applied repeatedly are foam fractionation (Davis et al. 2001; Noah et al. 2002), 
ultrafiltration (Sen and Swaminathan 2005), and adsorptiondesorption processes on polystyrene resins or activated carbon (Reiling et al. 1986; Dubey et al. 2005) as well as crystallization (Desai and Banat 1997). Discontinuous methods are acid or ammonium sulfate precipitation as well as organic solvent extraction. In most cases, the toxicity of the solvents does not allow repeated operation (Kuyukina et al. 2001; Mukherjee et al. 2006).

In this paper, we describe a method, which harvests the liquid boundary layer containing the adsorbed surfactants from the surface of culture broth. This surface layer of the culture is compressed in the neck of the flask. Then it is automatically harvested from the bottleneck and collected for further analysis.

The shape of the glass flask which is called "flounder" is shown in Fig. 1. In operation mode, there are two alternative positions. The flask is fixed in a steel cage, which automatically changes between these two positions. In the initial state, the flask is in a horizontal position allowing for surfactant adsorption (at the left side in Fig. 1). The surface-active substances adsorb at the large fluid interface. A rotating stirrer bar is supporting the mass transfer from bulk liquid to the surface. The following position of harvesting is shown at the right side of Fig. 1. When the flask is turned into a vertical position, the thickness of the layer containing the adsorbed surfaceactive substances increases by decreasing the surface area. It becomes possible to harvest this layer by sucking off the surface area with the help of a fine capillary (Lunkenheimer et al. 1987). The extract is collected in a storage flask. The time for adsorption can be set by the user as well as the time interval of suction and the number of harvesting cycles. The storage part (at the lower right side of the flask) ensures a constant liquid level in the absorption part over a

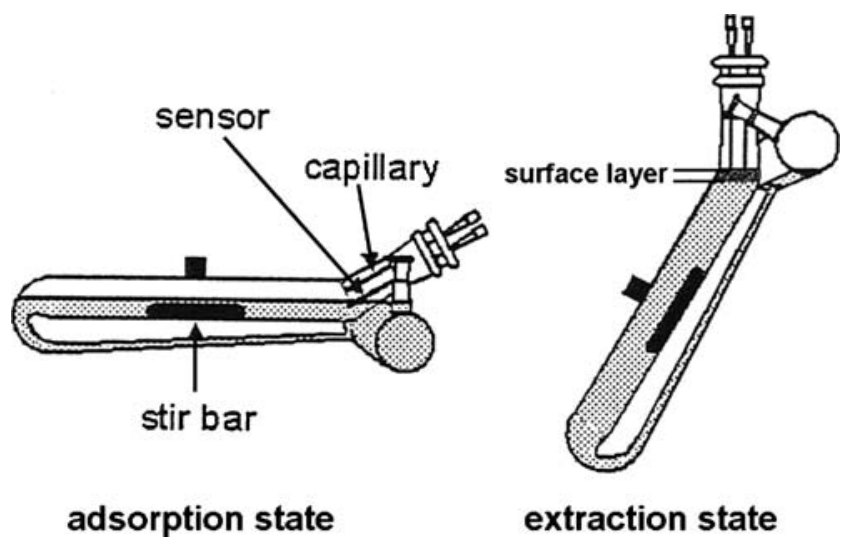

Fig. 1 Working principle of the "Flounder": In the adsorption state, surface-active substances are concentrated at the surface. In the harvesting state, the surfactant-enriched surface layer is harvested through a fine capillary (scheme adopted from (Lunkenheimer et al. 2004) certain number of cycles. A cycle is finished when both the adsorption and the harvesting state were passed.

This method was successfully applied for the removal of surfactant contaminations in solutions (Lunkenheimer 2002; Prosser et al. 2004) and the isolation of the surface film of human bronchoalveolar lavage fluid (Winsel et al. 2004). In the project described in this paper, the ability to remove efficiently the biosurfactant fengycin from cell-free culture centrifugate as well as broth from $B$. subtilis cultures is investigated.

Fengycin is a lipopeptide antibiotic produced by $B$. subtilis (Stein 2005). It has a broad spectrum of antifungal activities. Fengycin inhibits the growth of a wide range of plant pathogens and has a great potential as food preservative and plant protection agent. Despite this fact, the industrial use of fengycin is still limited. The main reasons are (1) the yield of fengycin is relatively low and (2) the purification methods are complex (Vanittanakom and Loeffler 1986; Ongena et al. 2005; Sun et al. 2006). A main problem is the separation of fengycin homologs and homologs of the polyketide antibiotic bacillaene, which are also synthesized by fengycin producing microorganisms. The possibility to separate these surface-active by-products using the flounder technique is described in this paper. The results of biosurfactant removal with the presented method are compared with results from experiments, where foam fractionation is applied.

\section{Materials and methods}

Strain and culture conditions

The strain B. subtilis DSM 21393 was used throughout this study. B. subtilis was maintained on an agar-solidified medium containing $15.6 \mathrm{~g} / \mathrm{l}$ peptone, $2.8 \mathrm{~g} / 1$ yeast extract, $1 \mathrm{~g} / 1$ glucose, and $5.6 \mathrm{~g} / 1 \mathrm{NaCl}$ at $4{ }^{\circ} \mathrm{C}$. Prior to experiments, B. subtilis cultures were incubated in shake flasks in a liquid medium containing $30 \mathrm{~g} / \mathrm{l}$ malt extract and $50 \% v / v$ potato extract (made from fresh potatoes) at $24{ }^{\circ} \mathrm{C}$ on a rotary shake at $150 \mathrm{rpm}$ for $48 \mathrm{~h}$ (all chemicals were purchased from Carl Roth GmbH, Karlsruhe, Germany, if not mentioned otherwise).

Removal of surface-active substances with the flounder

The flounder (Halcyonics GmbH, Göttingen, Germany) was operated with 440 to $480 \mathrm{ml}$ of supernatant or cell broth from flask cultures, which had entered the late exponential growth phase. The flounder was operated with an adsorption time of $60 \mathrm{~s}$ at a stirrer speed of $100 \mathrm{rpm}$ and a concentrate removal time of $480 \mathrm{~ms}$. When the level of 
the liquid volume in the flounder had fallen below the tip of the suction needle, cell-free centrifugate or culture broth was refilled. This limiting state is usually reached at cycle numbers of about 400 . The refill of another $40 \mathrm{ml}$ of original solution results in a small increase in biosurfactant concentration. This increase leads to a slight shift backwards in the separation characteristics of the equilibrium surface tension in dependence of the harvesting cycles. The extent of this shift can be estimated comparing the separation characteristics before and after the refill. Experiments were stopped when no fengycin was detectable in the collected fractions anymore.

Foam fractionation

Cells were removed from the culture in the late exponential growth phase by centrifugation at $10,000 \times \mathrm{g}$ for $20 \mathrm{~min}$. Foam was obtained by strong aeration of $100 \mathrm{ml}$ of cell-free centrifugate through a sinter plate at the bottom of a cylindrical flask. The foam was sucked from the top of the cylinder with a vacuum pump and fractionated in $10 \mathrm{ml}$ aliquots.

\section{Determination of surface tension}

The surface tension was measured with the semiautomated tensiometer K10T (Krüss GmbH, Hamburg, Germany) applying the de Noüy ring method. Measurements were performed at a constant temperature of $24{ }^{\circ} \mathrm{C}$. The sample vessel had a diameter of $75 \mathrm{~mm}$, which was appropriate for the experiments (Lunkenheimer and Wantke 1981). The surface tension of concentrate was analyzed whenever a total volume of $10 \mathrm{ml}$ of harvested solution was collected from the flounder. Surface tension in the flounder was measured whenever $10 \mathrm{ml}$ fraction was completed. Prior to surface tension measurement, all samples were diluted one fold with double-distilled water to reach a satisfying immersion depth for the ring.

\section{Quantification of fengycin and bacillaene}

For the purification of fengycin and bacillaene, cells were removed from the culture by centrifugation at $10,000 \times g$ for $20 \mathrm{~min}$. The surface active substances were adsorbed on an Amberlite $^{\circledR}$ XAD-16 resin (Sigma-Aldrich Inc., Munich, Germany). Adsorbed substances were eluted stepwise with $40 \%, 60 \%, 80 \%$, and $100 \%(v / v)$ methanol/water, respectively. The eluate used for further processing $(80 \% \mathrm{v} / \mathrm{v}$ methanol) was concentrated in a rotary evaporator. The residue was redissolved in pure methanol. The substances were quantified in a HP 1090 Liquid Chromatograph System (Hewlett Packard Inc., Palo Alto, CA) equipped with a UV/visible diode array detector. As separation column, a pursuit ${ }^{\circledR}$ XRs $\mathrm{C} 18$ column with a size of $150 \times$ $4.6 \mathrm{~mm}$ (Varian Deutschland GmbH, Darmstadt, Germany) was applied. The flow rate was held constant at $1 \mathrm{ml} / \mathrm{min}$. As eluent, gradients of acetonitrile and double distilled water of 100:0 v/v for $13 \mathrm{~min}, 10: 90 v / v$ for $13.1 \mathrm{~min}$, and 10:90 v/v for $15 \mathrm{~min}$ were used. Formic acid of $0.1 \% v / v$ was added to the eluents for improving high performance liquid chromatography (HPLC) analysis. The detection wavelength was set to $220 \mathrm{~nm}$. Peaks were integrated with the software package HP ChemStation, Revision A.05.01 (Hewlett Packard Inc., Palo Alto, CA).

Determination of adsorption parameters

In order to estimate the possibilities of the flounder to purify and/or separate the two different biosurfactants fengycin and bacillaene, it is useful to determine their adsorption parameters. The latter biosurfactants are usually derived from experimental equilibrium surface tension $\sigma_{\mathrm{e}}$ vs. the logarithm of the surfactant concentration $c$ isotherms of the surfactant. To assess the adsorption parameters of the two species, their experimental $\sigma_{\mathrm{e}} \mathrm{vs} . \log c$ isotherms were evaluated by the Szyszkowski-Langmuir adsorption equations (Lunkenheimer and Hirte 1992):

$\Delta \sigma_{\mathrm{e}}=R T \Gamma_{\infty} \times \ln \left(1+c / a_{\mathrm{L}}\right)$

and

$\Gamma=\Gamma_{\infty} /\left(1+a_{\mathrm{L}} / c\right)$.

where $\Gamma$ and $\Gamma_{\infty}$ denote the surface concentration and saturation surface concentration, respectively. The parameter $a_{\mathrm{L}}$ represents the surfactant concentration, at which the surfactant's adsorption layer has reached the state of half saturation.

The standard free energy of adsorption $\Delta G_{0}{ }^{\text {ad }}$ was calculated as:

$\Delta G_{0}^{\mathrm{ad}}=R T \ln a_{\mathrm{L}} / c_{n}$

in which $c_{n}=1 \mathrm{~mol} / \mathrm{dm}^{3}$ is used as standard concentration.

Data treatment

Curve fitting (splines) was performed using Matlab Version 7.0.1 (Mathworks Inc., Nattick, MA). To describe the efficiency of the method, the partition coefficient $K$ was calculated as follows:

$K=\frac{\text { Concentration in harvested fraction }}{\text { Concentration in bulk liquid }}$

The partition coefficient for fengycin will be denoted $K_{\mathrm{F}}$, for bacillaene, it will be denoted $K_{\mathrm{B}}$. 


\section{Results}

Adsorption parameters of fengycin and bacillaene

The resulting experimental dependences of $\sigma_{\mathrm{e}}$ and $\log c$ are presented in Fig. 2. The adsorption parameters derived from Eqs. 1a and $1 \mathrm{~b}$ as well as the free energy of adsorption derived from Eq. 2 are summarized in Table 1. Even though they are calculated from a certain section of the whole experimental $\sigma_{\mathrm{e}}$ vs. $\log c$ isotherm without further surface purification, they should represent a close approximation to the exact adsorption data. The data represent values containing reasonable molecular information, since they well reflect the comparatively big molecular size of the biosurfactants.

The surface activity of fengycin is greater than that of bacillaene by almost two orders of magnitude. The ratio of $a_{\mathrm{L}}$ is:

$r=\left(a_{\mathrm{L}}\right)_{\text {feng }} /\left(a_{\mathrm{L}}\right)_{\mathrm{bac}}=1.39 \times 10^{-10} / 2.76 \times 10^{-8} \cong 1 / 50$

Hence, at an equal adsorption at the air/water interface, only $1 / 50$ of the concentration of fengycin as compared to that of bacillaene is needed. In good accordance to these findings, the free energy of adsorption is lower for fengycin $(-12.6 \mathrm{~kJ} / \mathrm{mol})$.

Separation of biosurfactants from cell-free centrifugate

Separation of surface-active substances was studied using cell-free centrifugate from flask cultures after $93 \mathrm{~h}$ of growth (early stationary phase). In Fig. 3, the time course development of fengycin concentration in the fractions as well as in the remaining solution in the flounder is shown. During the first 200 harvesting cycles, $K_{\mathrm{F}}$ equals 4 . After

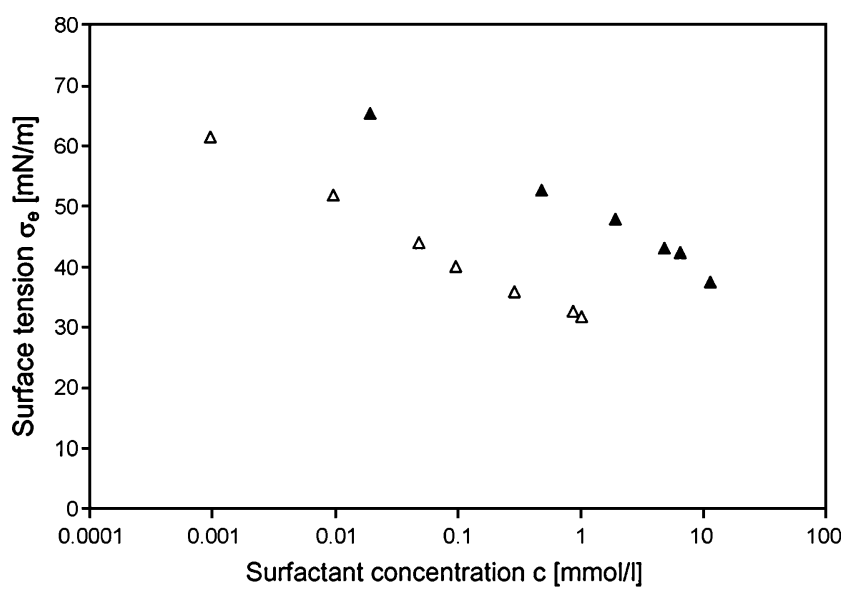

Fig. 2 Isotherms of experimental equilibrium surface tension $\sigma_{\mathrm{e}}$ of fengycin (open triangle) and bacillaene (close triangle) in dependence of their concentration
Table 1 Overview of adsorption parameters of fengycin and bacillaene estimated using Eqs. 1a, 1b, and 2 based on the surface tension measurements in Fig. 2

\begin{tabular}{lll}
\hline Parameter [unit] & Fengycin & Bacillaene \\
\hline$a_{\mathrm{L}}\left[\mathrm{mol} / \mathrm{dm}^{3}\right]$ & $1.39 \times 10^{-10}$ & $2.76 \times 10^{-8}$ \\
$\Gamma_{\infty}\left[\mathrm{mol} / \mathrm{m}^{2}\right]$ & $1.99 \times 10^{-6}$ & $2.31 \times 10^{-6}$ \\
$A\left[\AA^{2} / \mathrm{molecule}\right]$ & 83.4 & 71.9 \\
$\Delta G_{0}[\mathrm{~kJ} / \mathrm{mol}]$ & -65.2 & -52.6 \\
\hline
\end{tabular}

350 cycles, $50 \%(w / w)$ of the original amount of fengycin within the centrifugate is collected in the harvested samples. During further harvesting, the fengycin concentration difference between fractions and centrifugate decreases to $0.003 \mathrm{mmol} / 1$ after 800 cycles. At this state, $95 \%(w / w)$ of the initial amount of fengycin is removed. Thus, the optimal operation mode for fengycin removal is at a bulk concentration above $0.0015 \mathrm{mmol} / \mathrm{l}$ during the first 250 harvesting cycles.

The separation of substances generally depends on their surface activity. When comparing fengycin and bacillaene, the portion of fengycin is greater in the fractions throughout the whole experiment (Fig. 4). At the onset of the experiment, substances with an even greater surface activity than fengycin and bacillaene might be adsorbed. Hence, the separation of the two substances is not accomplished at this point. The maximum separation is reached after almost 500 cycles. Bacillaene becomes unstable when fengycin and other surface-active substances are removed from the culture: Its concentration falls below $0.002 \mathrm{mmol} / \mathrm{l}$ after 475 cycles, even though samples were protected against UV radiation. At the onset of the experiment, the initial concentration of bacillaene is $0.2 \mathrm{mmol} / \mathrm{l}$. In fractions following 475 cycles, the bacillaene concentration is nearly undetectable, while the bacillaene concentration in the solution in the flounder remains stable. Hence, the complete

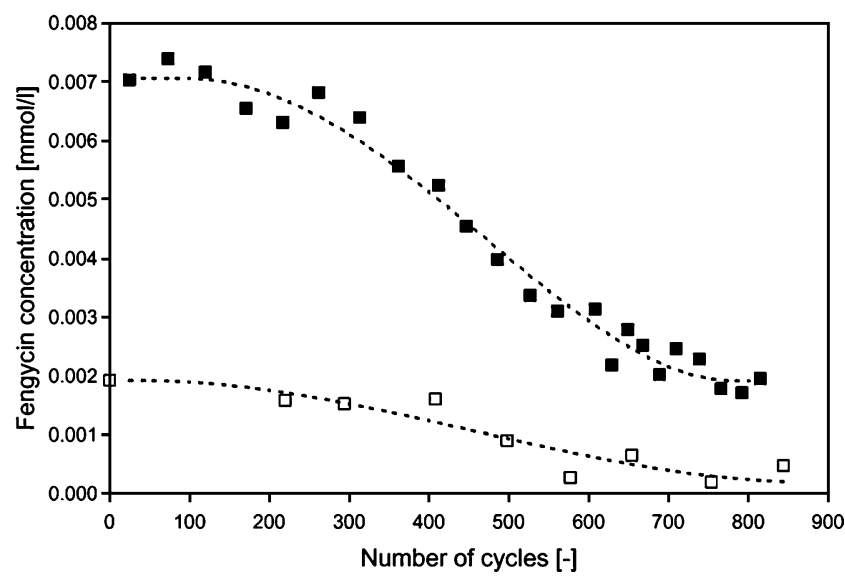

Fig. 3 Fengycin concentration in the cell-free centrifugate (open square) and in the fractions harvested with the flounder (close square). Dashed lines represent curve fits 


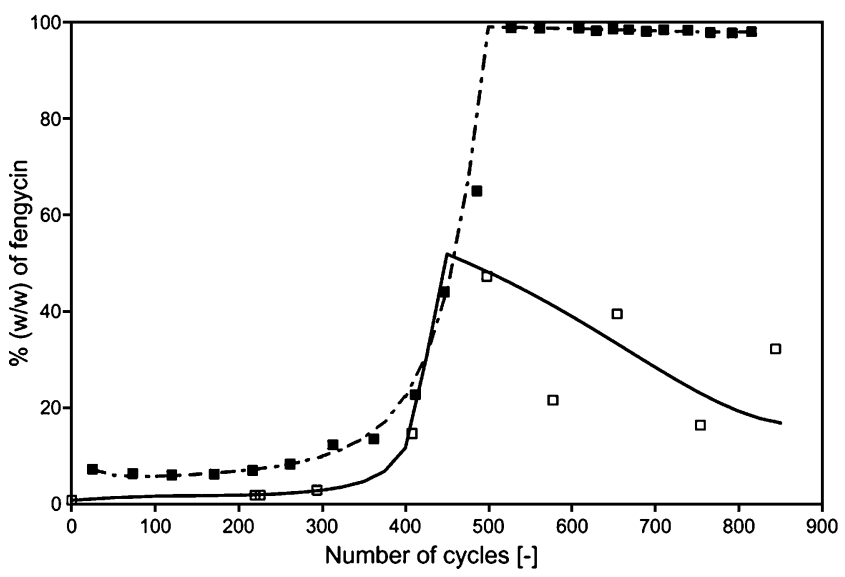

Fig. 4 Portion of fengycin in the total amount of fengycin and bacillaene $(\% w / w)$ in cell-free centrifugate (open square) and in fractions harvested with the flounder (close square). Lines represent curve fits

separation of fengycin from bacillaene is achieved after 475 cycles.

\section{Separation of biosurfactants from culture broth}

When fengycin is not extracted from cell-free centrifugate but from culture broth, the concentration increase in the fractions is slightly lower. While $K_{\mathrm{F}}=4$ during the first 500 cycles in the experiments with the centrifugate, $K_{\mathrm{F}}=3.5$ when using culture broth containing bacterial cells. The partition coefficient for bacillaene, $K_{\mathrm{B}}$, is not severely influenced, remaining below 1 when using centrifugate or culture broth.

\section{Change of surface tension during separation}

In Fig. 5, the dependence of the equilibrium surface tension values in the fractions together with the corresponding values of the bulk solutions in the flounder is shown.

Prior to cultivation, the medium had a surface tension value of $52 \mathrm{mN} / \mathrm{m}$, which indicates the presence of surfaceactive material. After cultivation, the surface tension dropped below $30 \mathrm{mN} / \mathrm{m}$. Such a low value indicates saturation of the solution's adsorption layer, possibly already at a bulk concentration greater than its critical concentration of micelle formation.

The dependence of $\sigma_{\mathrm{e}}(j)$ of the bulk solution in the flounder reveals at least two distinct plateau values with rising number of harvesting cycles. The feature of various plateaus in the function $\sigma_{\mathrm{e}}(j)$ is striking. This behavior is characteristic of the effect of surface-active trace impurities, usually observed with solutions of "as received surfactants". The increase in $\sigma_{\mathrm{e}}(j)$ up to a following plateau value is due to the successive reduction of the bulk concentration of a surface-active component. This component has the relatively greatest surface activity but a small concentration as compared to that of the main surfactant (Lunkenheimer and Miller 1987).

The removal of the adsorption layer from the surface results also in a small but detectable reduction of the trace impurity's concentration in the bulk solution remaining in the flounder. When its concentration has become negligible and/or sufficiently small as compared to that of the main surfactant, it will no longer influence the adsorption properties in this particular processing stage. Between 220 $<j<475$ cycles, another similar surface behavior followed consisting of an increase of the $\sigma_{\mathrm{e}}$ value and amounting into the second plateau value given by $\sigma_{\mathrm{e}}=31.0 \mathrm{mN} / \mathrm{m}$. It is evident that a further surfactant component had been removed via adsorption during this interval. During the following processing stage, the component having the next greatest surface activity is preferentially adsorbed as long as the interval is characterized by a constant $\sigma_{\mathrm{e}}$ value. This state is related to the narrow interval between $450>j>475$ cycles. It becomes obvious that on principle, it is possible that additional intervals with analogous constant plateau values might be observed, provided the biosurfactant solution's boundary conditions would hold correspondingly. Within the last processing stage for $j>475$ cycles, the most effective increase in the corresponding surface tension values occurs, characterized by the steepest slope in $d \sigma_{\mathrm{e}} / d \ln c$. The surface tension of the solution in this interval does approach another constant $\sigma_{\mathrm{e}}$ value of $33.4 \mathrm{mN} / \mathrm{m}$.

The initial $\sigma_{\mathrm{e}}(j)$ dependence in experiments with centrifugate and culture broth containing the same initial concentration of fengycin does not show differences to the behavior in Fig. 5. The time course of the surface tension within the bulk culture broth is decreasing during the first 300 cycles. The surface tension of the culture broth becomes lower than in the centrifugate. The difference

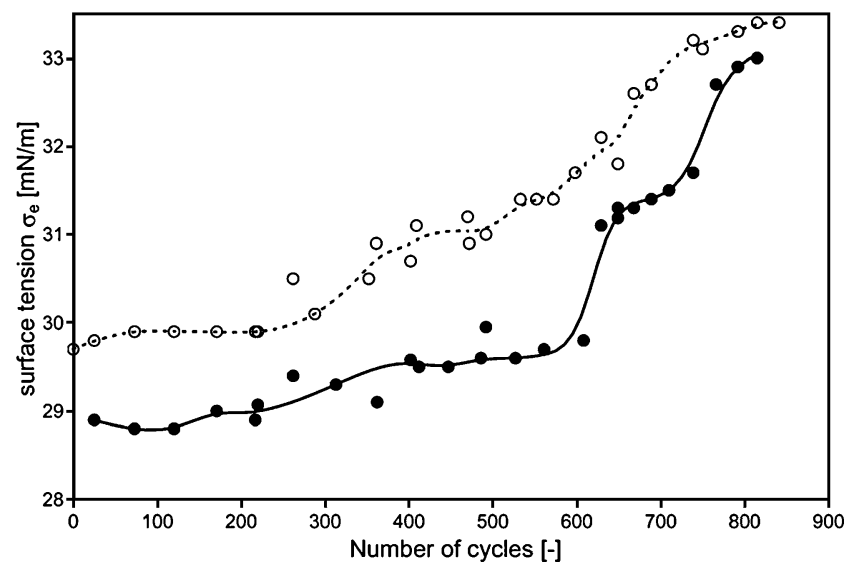

Fig. 5 Surface tension $\sigma_{\mathrm{e}}$ in the fractions (close circle) and in the bulk of the cell-free centrifugate in the flounder (open circle). Lines represent curve fits 
increases up to $1.5 \mathrm{mN} / \mathrm{m}$ during the experiments. From optical density measurements and microscopic observations, no cell lysis could be detected. Since the cells in the flounder are still viable, it is possible that additional surface-active substances are secreted.

Purification of surfactant-enriched fractions

Figure 6 shows the HPLC chromatograms (spectra) of following samples: (a) a centrifugate of culture broth obtained in the early stationary phase, (b) a fraction harvested with the flounder after 100 cycles, (c) a sample from the solution in the flounder after 100 cycles, and (d) the first fraction obtained using foam fractionation, respectively. All samples had been prepared for HPLC analysis as described in the "Materials and methods" section.

The chromatogram (a) shows that the first bulk sample in the flounder at the beginning of the experiment contains various substances. The peaks at 8.1 and $8.4 \mathrm{~min}$ are bacillaene, the peaks between 6.9 and $7.9 \mathrm{~min}$ are fengycin
Fig. 6 Peaks of fengycin (open triangle) and bacillaene (close triangle) in HPLC spectra of cell-free centrifugate of culture broth at the early stationary phase (a), after a following treatment with 100 harvesting cycles (c), of the collected fraction after 100 harvesting cycles (b), and of the first foam fraction (d)

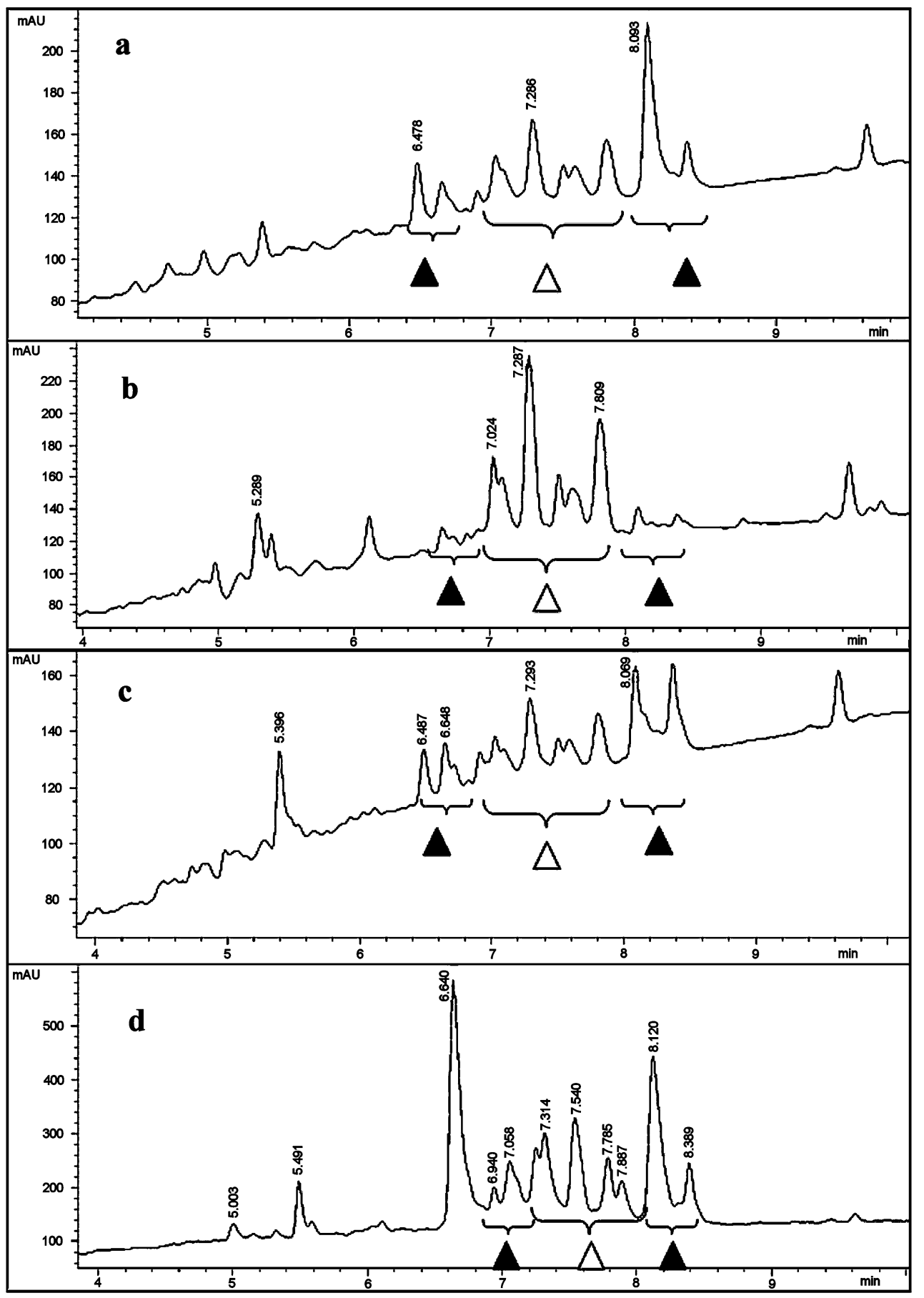


homologs, and the peaks between 4 and $6.5 \mathrm{~min}$ are other lipopeptides, respectively. The first fraction removed from the flounder after 100 cycles (b) contained fengycin and other lipopeptides in a range at 4 and 7 min only. The peaks of bacillaene are barely visible. Compared with the first fraction, the spectrum of the bulk solution after 100 cycles (c) shows several different peaks (similar to the spectrum a). Both bacillaene peaks are clearly visible on this spectrum.

\section{Foam fractionation}

Figure 7 shows the concentration of fengycin and bacillaene in the foam fractions obtained using foam fractionation. Seven fractions were collected using this method. The highest concentration of fengycin and bacillaene is detected in the first fraction. However, compared to Fig. 4, the fengycin portion in the first fraction is only $1.87 \%$ of the total fengycin and bacillaene amount. In the second and third fractions, the concentrations of fengycin and bacillaene are substantially lower. In the fourth and subsequent fractions, both substances are absent. Concentrations in the first fraction are higher than in the fraction obtained with the flounder. However, the separation of fengycin from bacillaene by foam fractionation is not possible. The HPLC spectrum of the first foam fraction (d) in Fig. 6 contained both fengycin and bacillaene. The number of components in the first foam fraction (d) does not differ much from that in the bulk centrifugate (a).

\section{Discussion}

The flounder can be considered as a favorable tool for the treatment prior to further downstream processing or analysis. By applying this method, it is possible to collect

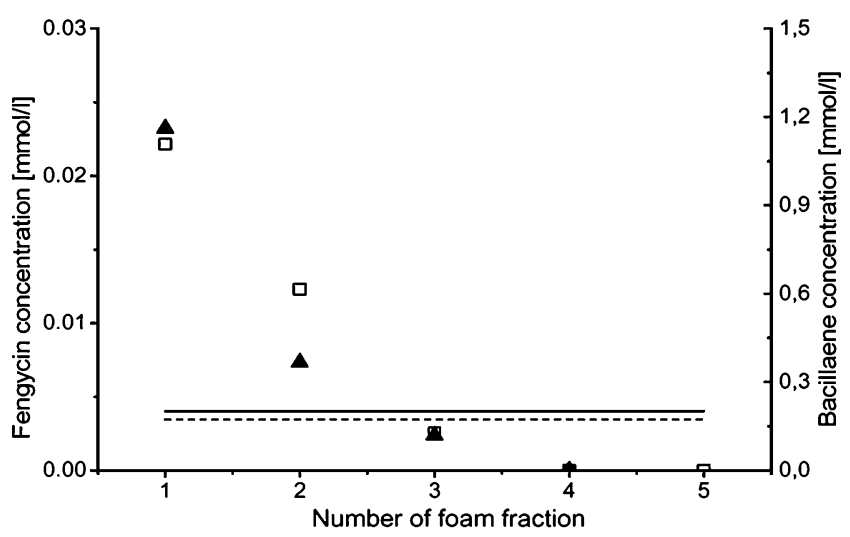

Fig. 7 Concentrations in the cell-free culture centrifugate and the first five foam fractions of fengycin (open square) and bacillaene (close triangle). The straight line indicates the fengycin concentration and the dashed line indicates the bacillaene concentration in the culture and concentrate fengycin from B. subtilis cultures at the onset of the stationary phase. A simultaneous purification with respect to bacillaene could be observed.

In order to understand the purification characteristic of the biosurfactant system under consideration, it is important to consider the concentration ratio of all components competing for adsorption together with respect to their surface activities (Eqs. 1a and 1b). Thus, it is the ratio $c_{i} / a_{\mathrm{L}, i}$ of each surface-active component $i$ that determines the surface behavior, but not necessarily its bulk behavior.

Generally, when solutions of "as received surfactants" are purified by the flounder technique to get the required grade of "surface-chemical purity" their purification characteristic $\sigma_{\mathrm{e}}(j)$ reaches finally one plateau value $\sigma_{\mathrm{e}}=$ constant. In this state, there will be no longer any falsifying influence of any surface-active impurity. The adsorption properties of this solution are entirely determined by its bulk concentration (Lunkenheimer and Miller 1987). Two peculiarities have to be taken into consideration at the system described in this study. Firstly, the biosurfactants have much greater surface activities than those of usually applied synthetic surfactants, such as sodium dodecyl sulphate. Secondly, several surface-active substances are present at low concentrations. This, in turn, means that their effect on the adsorption behavior should rather be considered in terms of surface-active trace impurity effects only, without any accompanying main surfactant component of relatively much bigger bulk concentration. The initial dependence of $\sigma_{\mathrm{e}}(j)$ indicated the presence of a surfaceactive component of possibly extreme surface activity and extremely small concentration. It had been removed from the solution during the harvesting process in the initial state between $0 \leq j \leq 80$ cycles. However, several other surfaceactive components are left in the bulk solution in the intermediate processing state between $80<j<220$ cycles. Thus, a constant $\sigma_{\mathrm{e}}$ value of the solution in the latter state is related to the overall effect of all residual surface-active components still present in the bulk solution. The reason why there appears a second plateau, and maybe even further additional ones in the dependence of $\sigma_{\mathrm{e}}(j)$, lies in the above mentioned peculiar boundary conditions of this biosurfactant system. As long as the solution's $\sigma_{\mathrm{e}}$ value is constant, the adsorption will be mainly determined by the surfactant of greatest surface activity and a relevant concentration. Therefore, this component will be preferentially adsorbed. The repeated removal of the adsorption layer leads to a decrease of its concentration (i.e., for $j>200$ cycles). It will finally completely loose its influence on the adsorption layer. Now, the substance with the highest surface activity and relevant concentration among the remaining components will determine the $\sigma_{\mathrm{e}}$ value of the solution.

The development of $\sigma_{\mathrm{e}}$ of the harvested fractions in Fig. 5 resembles that of the sucked off solutions. The 
corresponding absolute surface tension values of the process' $\sigma_{\mathrm{e}}(j)$ dependence are always a little lower. This is due to the fact that the component of greater surface activity will always preferentially be enriched in the adsorption layer. On this background, it is of extraordinary importance that the surface tension of the solution in the flounder and the harvested fractions becomes identical. At the final processing stage, there is only one single surface-active component left in the solution of the flounder as well as in the final fraction of the harvesting process. Hence, the final plateau value of $\sigma_{\mathrm{e}}=33.4 \mathrm{mN} / \mathrm{m}$ does correspond to the surface chemically pure solution of the one single surfactant component left after the entire purification procedure via adsorption. This conclusion does hold generally as long as the components taking part in the competing adsorption process differ by their surface activity and/or bulk concentration.

The harvesting of the biosurfactant's adsorption layers is an alternative approach to common extraction procedures. Since the utilization of the flounder does not include any solvents, the method can be assumed to be cost effective compared to liquid extraction systems. The partition coefficient $K$ for lipopeptides in aqueous two-phase systems ranges between 2 and 5 (Drouin and Cooper 1992; Kuboi et al. 1994; Sinha et al. 2000). These are comparable values to flounder experiments. In contrast to two-phase systems, no phase partitioning compounds are necessary. The presence of cells does only slightly influence the separation efficiency in flounder experiments, which is in contrast to extraction procedures, where cells can halve the mass transfer (Pursell et al. 2004).

While the partition coefficient of foam fractionation is comparable to the coefficients in flounder experiments, the separation of fengycin and bacillaene can not be obtained with foaming. While the partition coefficient of fengycin $K_{\mathrm{F}}=4$ when using centrifugate, the partition coefficient for bacillaene $K_{\mathrm{B}}=0.55$. In the foam fractionation experiments, $K_{\mathrm{F}}=5.5$, while $K_{\mathrm{B}}=6.7$. Hence, the ratio of partition coefficients for fengycin and bacillaene is ninefold higher at the treatment with the flounder. The thickness of the harvested surface layer in the flounder is approximately $0.4 \mathrm{~mm}$, while in our experiments $8 \mathrm{~mm}$ of the upper layer of a sample are needed to obtain a foam fractionation. Hence, all compounds in and near the surface layer of the liquid are separated and the method does not favor the separation between the surface-active components.

Since the presence of cells does not essentially disturb the extraction process, the removal of surfactants directly from cultures is possible. The treatment of cell containing cultures could be valuable, when the synthesis of biosurfactants is subject to product inhibition. Additionally, in screening processes, the flounder can be a valuable alternative whenever low surfactant bulk concentrations lead to difficulties in further analytical procedures.
Summarizing the application of the flounder technique, it is possible to prepare surfactant solutions in a required grade ensuring "surface-chemical purity" of their adsorption layers. However, as this technique is based on the fundamentals of adsorption applied under well-defined boundary conditions, it is sure that also the removal of the impurity components is being accomplished by following well-defined thermodynamic relationships. Hence, the favorable possibility of stepwise separating these components is only the reasonable consequence of this process.

The effectiveness of separation of different biosurfactants can be further increased by applying submicellar concentrations. Analytical identification together with relevant adsorption properties of the various surface-active substances becomes favorably possible.

Limitations of the presented method are reached, when larger volumes should be treated. So far, it is possible to remove biosurfactants from cultures in the laboratory scale using the flounder. The utilization of this principle in larger scaled processes or for continuous removal of biosurfactants will be the focus of following studies.

Open Access This article is distributed under the terms of the Creative Commons Attribution Noncommercial License which permits any noncommercial use, distribution, and reproduction in any medium, provided the original author(s) and source are credited.

\section{References}

Banat IM, Makkar RS, Cameotra SS (2000) Potential commercial applications of microbial surfactants. Appl Microbiol Biotechnol 53:495-508

Davis DA, Lynch HC, Varley J (2001) The application of foaming for the recovery of Surfactin from B. subtilis ATCC 21332 cultures. Enzyme Microb Technol 28:346-354

Desai JD, Banat IM (1997) Microbial production of surfactants and their commercial potential. Microbiol Mol Biol Rev 61(1):47-64

Drouin CM, Cooper DG (1992) Biosurfactants and aqueous two-phase fermentation. Biotech Bioeng 40:86-90

Dubey KV, Juwarkar AA, Singh SK (2005) Adsorption-desorption process using wood-based activated carbon for recovery of biosurfactant from fermented distillery wastewater. Biotechnol Prog 21:860-867

Kuboi R, Maruki T, Tanaka H et al (1994) Fermentation of Bacillus subtilis ATCC 6633 and production of subtilin in polyethylene glycol/phosphate aqueous two-phase systems. J Ferment Bioeng 78(6):431-436

Kuyukina MS, Ivshina IB, Philp JC et al (2001) Recovery of Rhodococcus biosurfactants using methyl tertiary-butyl ether extraction. J Microbiol Methods 46:149-156

Lunkenheimer K (2002) On the problem of surface-chemical purity of surfactants-phenomena, analysis, results, consequences, and prospects. Encyclopedia of surface and colloid science. Dekker, New York, pp 3739-3772

Lunkenheimer K, Hirte R (1992) Another approach to a surface equation of state. J Phys Chem 96:8683-8686

Lunkenheimer K, Miller R (1987) A criterion for judging the purity of adsorbed surfactant layers. J Colloid Interface Sci 120:176-183 
Lunkenheimer K, Pergande H-J, Krüger H (1987) Apparatus for programmed high-performance purification of surfactant solutions. Rev Sci Instrum 58:2313

Lunkenheimer K, Wantke K-D (1981) Determination of the surface tension of surfactant solutions applying the method of lecomte du noüy (Ring-Tensiometer). Colloid Polymer Sci 259:354

Lunkenheimer K, Wienskol G, Prosser AJ (2004) Automated highperformance purification of surfactant solutions: study of convective-enhanced adsorption. Langmuir 20(14):5738-5744

Makkar RS, Cameotra SS (2002) An update on the use of unconventional substrates for biosurfactant production and their new applications. Appl Microbiol Biotechnol 58:428-434

Mukherjee S, Das P, Sen R (2006) Towards commercial production of microbial surfactants. Trends Biotechnol 24(11):509-515

Noah KS, Fox SL, Bruhn DF et al (2002) Development of continuous surfactin production from potato process effluent by Bacillus subtilis in an airlift reactor. Appl Biochem Biotech 98-100:803-813

Ongena M, Jacques P, Toure Y et al (2005) Involvement of fengycintype lipopeptides in the multifaceted biocontrol potential of Bacillus subtilis. Appl Microbiol Biotechnol 67(5):692-698

Peypoux F, Bonmatin JM, Wallach J (1999) Recent trends in the biochemistry of surfactin. Appl Microbiol Biotechnol 51:553-563

Prosser AJ, Retter U, Lunkenheimer K (2004) On the adsorption kinetics of surface-chemically pure n-dodecanoic acid at the air/ water interface. Langmuir 20:2720-2725
Pursell MR, Mendes-Tatsis MA, Stuckey DC (2004) Effect of fermentation broth and biosurfactants on mass transfer during liquid-liquid extraction. Biotech Bioeng 85(2):155-165

Reiling HE, Thanei-Wyss U, Guerra-Santos LH et al (1986) Pilot plant production of rhamnolipid biosurfactant by Pseudomonas aeruginosa. Appl Environ Microbiol 51:985-989

Sen R, Swaminathan T (2005) Characterization of concentration and purification parameters and operating conditions for the smallscale recovery of surfactin. Process Biochem 40:2953-2958

Sinha J, Dey PK, Panda T (2000) Aqueous two-phase: the system of choice for extractive fermentation. Appl Microbiol Biotechnol 54 (4):476-486

Stein T (2005) Bacillus subtilis antibiotics: structures, syntheses and specific functions. Mol Microbiol 56(4):845-857

Sun L, Lu Z, Bie X et al (2006) Isolation and characterization of a coproducer of fengycins and surfactins, endophytic Bacillus amyloliquefaciens ES-2, from Scutellaria baicalensis Georgi. World J Microbiol Biotechnol 22:1259-1266

Vanittanakom N, Loeffler W (1986) Fengycin - a novel antifungal lipopeptide antibiotic produced by Bacillus subtilis F-29-3. J Antibiot 39(7):888-901

Winsel K, Lunkenheimer K, Geggel K et al (2004) Automatic adsorptive isolation and biophysical and biochemical characterization of the surface film of human bronchoalveolar lavage fluid. Tenside Surfactants Deterg 41(1):10-18 